\title{
Analysis of the Tool Support for Business Model Innovation
}

\author{
Sabine Klein \\ German Research Center for Artifi- \\ cial Intelligence \\ $\underline{\text { sabine.klein@dfki.de }}$
}

\author{
Andreas Emrich \\ German Research Center for Artifi- \\ cial Intelligence \\ andreas.emrich@dfki.de
}

\author{
Peter Fettke \\ German Research Center for Artifi- \\ cial Intelligence \\ peter.fettke@dfki.de
}

\begin{abstract}
Organizations adapt their business models (BM) frequently to remain competitive. One reason for this is the digitalization of products and services. A change in strategy often brings along an adaption of the BM or a complete business model innovation (BMI). In this context, software tools can support users in the presentation and analysis of their BM by providing methodological knowledge. The assessment of a software tool is influenced by different orientations and functionalities, e.g., for pure BM representation or simulation. This contribution provides a procedure for systematically assessing BMI tools and offers an overview of existing BM tools as well as their support for BMI and a related transformation process. The results show that early phases of BMI are well supported, while later phases are hardly represented. A possible reason for this lies in the complexity of later BMI phases. First steps towards supporting later phases through software are discussed.
\end{abstract}

\section{Introduction}

Many organizations continuously innovate their processes, products and services. Nevertheless, there are large companies which have not survived digitalization despite their innovative strength. Their failure usually has multiple causes but still, a continuous business model (BM) improvement and innovation ensures longterm success [1]. Larger enterprises spend approx. 10\% of their innovation budget on creating new BM, while this percentage is much lower for small and mediumsized companies [2]. The BM of companies such as Google or Amazon are acknowledged worldwide due to their focus on integrated solutions comprising products and services. This affects all dimensions of value creation, from marketing and sales, to product development and pricing models [3]. Business Model Management (BMM) supports all activities related to the initial design, implementation, modification, controlling, adaptation and improvement of $\mathrm{BM}$ over time. To implement and manage BM successfully, software tools seem essential as operations such as simulation or meaningful visualization are not feasible without software support [4]. In many cases BM tools or methods are only used to depict the current BM and to serve as a reporting or governance tool at one point in time, e.g., for decision boards. Since the topic of BM has been an integral part of research for over 20 years, work on software support has been ongoing throughout this period. Several researchers emphasize that BM tools can help organizations to design, develop and innovate their BM better [5]. Within literature, tools for BM are considered to facilitate the construction and innovation of $\mathrm{BM}$ by providing modellers and users with information and guidance through the process. Advantages over a merely paper-based application include editing functions, model management or syntax checks [6]. Although many software tools are described in the literature or offered online, it is not obvious which modelling scenarios or objectives can be addressed with the tools and which phases of BMI are actually supported.

Against this background, the goal of this contribution is to introduce a procedure for assessing software support for BMI. For this purpose, we give an overview of current software tools for BM to shed light on the current state of development and to identify potentials for further extensions. Besides, the extent to which the tools support different modelling and adaptation scenarios closely linked to BMI is analysed. In our research, we specifically approach the following research questions:

- RQ1: Which software tools for BM exist and how can they be assessed?

- RQ1a: To what extent do these tools support the phases of BMI?

- RQ1b: In which way do the tools offer the support for the user?

- RQ2: Which modelling scenarios are covered with the help of the BM software tools?

The remainder of this paper is structured as follows: Section 2 puts the concepts of BMM and BMI into context and presents related literature for BM software 
tools. Section 3 elaborates on the research methodology we use to find and analyze existing BM tools as well as the criteria and procedure to evaluate them. Section 4 outlines the results of the analysis and addresses the research questions. We discuss the implications of our results as well as limitations in section 5 before we round off our work with a conclusion and an outlook to future research.

\section{Related Work}

\subsection{Business Model Management and Innova- tion}

BM present the core features of an enterprise and its products. They provide fundamental insights into innovations, business processes and routines, and are used to depict, optimize and evaluate the business goals of a company [7]. In this context BMM is an instrument for controlling the objectives of an organization. This comprises all target-oriented activities like the design, implementation, modification, adaptation and the control of a BM to generate and secure competitive advantages [8]. In literature, different phases of BMM beyond the design stage are described. These phases usually have a similar character like implementation, operation, change, controlling or analysis and management. BM in general can either be used as a kind of blueprint for the alignment of an organization respectively product or dynamically, by applying them as a tool for change and innovation in the company [9].

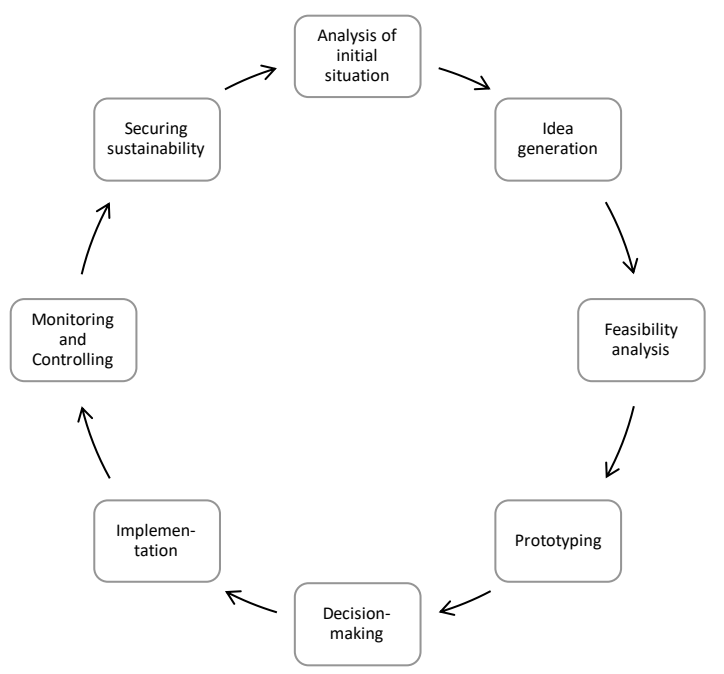

\section{Figure 1: Business model innovation process (based on [8])}

There is consensus that as a source of innovation, $\mathrm{BM}$ are important drivers for transformation and renewal [10], and an essential success factor for the sustainability of organizations [11]. BMI, as part of BMM, is linked to the emergence of new or heavily adapted BM. Drivers were and are mainly technological advancement, a dynamic market environment including competitors and changing customer requirements [3]. There exist different approaches to the BMI process (an overview is given by Wirtz et al. [12]). Wirtz describes a detailed BMI process (figure 1), which provides the criteria for the software tool analysis in section 3.3.

\subsection{Existing research in the field of BM tools}

Previous studies and overviews on BM software tools are also considered for the tool analysis in this contribution. For this purpose, a literature review [13] was conducted in the databases AIS eLibrary, IEEE Xplore, Scopus and Springer. The keywords business model and software tool ("business model" AND software tool) should be included in the title and/or the abstract. The search was limited to the years from 2000 onwards. The only criterion for a search result to be considered relevant was that a software tool, prototype, or overview of $\mathrm{BM}$ or BMI tools had to be presented. If a reference was made to another literature source for a software tool, it was also considered. The term 'tool support' in this contribution is used in the meaning of software support, whereby this includes method support in a broader sense.

\section{Table 1: Literature search for BM software tools}

\begin{tabular}{l|l|l}
\hline Database & \# results & Relevant results \\
\hline AIS eLibrary & 4 & {$[14],[15],[16],[17]$} \\
\hline IEEE Xplore & 39 & {$[18]$} \\
\hline Scopus & 43 & {$[19],[20]$} \\
\hline Springer & 29 & $\begin{array}{l}{[20],[21],[22],[23],[24],} \\
{[25],[26],[5],[27],[28] ;} \\
{[29],[6],[30],[31],[32]}\end{array}$ \\
\hline
\end{tabular}

The literature search yields 16 relevant results as well as 5 additional results found in the overview by [5] (table 1). The publication from IEEE Xplore [18] featured the so-called Business Model Builder, a digital twin of a BM. Although the authors describe a comprehensive method for creating and adapting a BM, the corresponding software tool seems to be unavailable. Unfortunately, this also applies to other tools found in the literature search (Assist is only available in connection with a consulting service [24], the BM-tool for additive manufacturing technologies is not freely available [19], OctoProz [17], Enterprise Architecture Analysis Tool (EAAT) [25], Software Business Model Tool [28], BM Wizard [29], BMeG editor [22]). Other tools, on the contrary, are only presented within literature, as they are still prototypes (Business Model Developer [26], BM development tool [30], BM engineering tool [31]). 
In both Scopus and Springer, a tool for testing the robustness of a BM was found. The tool started as a spread-sheet version [20], [21], and is now integrated in the online-tool Business Makeover, which is included in section 4. The search in the AIS Library yielded, among others, a study conducted by Szopinski et al. [14]. They analyze available free-of-charge online-tools for business modelling. The survey focuses on user interactions and technical aspects of the BM tools, such as navigation and filtering as well as the underlying architecture. The set of tools presented by the authors is also integrated in the tools to be investigated in sections 3 and 4 . Another study based on the one by Szopinski et al. analyzes the same BM tools with the Business Model Canvas (BMC) as the underlying method. They derive a taxonomy of the characteristic functions of the BM tools [23]. Szopinski also presents how individuals can be cognitively stimulated while generating BM ideas to help researchers build and evaluate software-based tools for BM creation [16]. In his work he refers to two concepts for BM software tools, namely a hybrid intelligence decision support system for BM validation [33] and a BM idea generation tool with prefilled BM [34]. Both prototypes are not available yet. Schoormann et al. describe early steps in implementing a Green Business Modelling editor (beta) [15], [27] to focus on the sustainability of BM. Fritscher et al. [6] use a prototype version of the $B M \mid D e s i g n e r$ [35] to analyse how users interact with a business modelling tool, which was also found in our online tool search. Another overview on BM methods (and some corresponding tools) is given by [32]. The authors focus on the origin as well as the visualization method and modelling language for BM.

Our work relies on related work and differs from it in the following ways: While [14], [23], [32] focus on the mere technical features or modelling languages of freely available BM software, we in contrast want to illustrate which content-related support of BMI can actually be provided by current tools. The tools can differ in functions to support the user during a BMI as this difference is irrelevant to the realization of a BMI project in our analysis. The literature search shows that there are efforts for tool-supported business modelling, but that these often do not go beyond the status of a prototype. To the best of our knowledge, an analysis of BM software tools regarding the support for the BMI process and related modelling scenarios seems not available yet.

\section{Research Method}

\subsection{Evaluation procedure}

The overall procedure for our tool evaluation is shown schematically in figure 2. First, the BMI phases were defined since different subdivisions and definitions of the phases exist in literature. For our analysis, the phases according to Wirtz [36] were used (section 2.1). In addition, sub-criteria per BMI phase are specified, which characterize each phase in more detail (section 3.3). Then a standard BMI use case was created that contains problems and goals for each BMI phase (section 3.3). At the same time, existing BM tools (section 3.2) are searched for. Also, the group of people who will test the different BM tools is formed (section 3.2). Subsequently, the actual evaluation of the tools is carried out with the predefined categories (section 4.2) before the results are compared (sections 4.2 and 4.3) and discussed (section 5). The procedure can be used for future $\mathrm{BM}$ tool comparisons and then also be carried out with existing use cases or an already selected BM tool, for example.

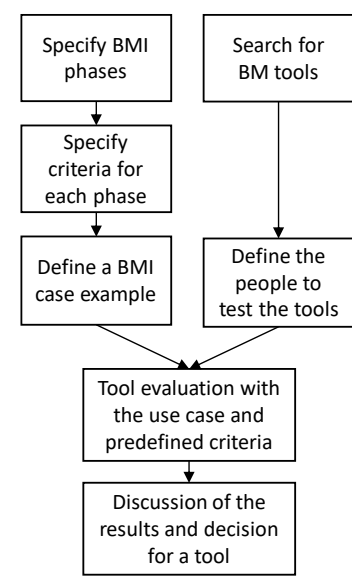

Figure 2: Procedure for evaluating BMI tools

\subsection{Search for BM tools}

To identify current available tools for innovating BM beyond the literature search, the search engines Google, DuckDuckGo and bing were used. The terms "software tool" and "business model" were entered in the simple search. As a next step, instead of "business model", the search terms "business model innovation" and "business model management" were entered. For every request, the first 100 results were searched for relevant tools or overviews on different BM tools. Additionally, the already existing tool overviews of the authors from section 2.2 [14], [20], [21] were integrated into the set of results. In this respect, the tools found are obtained from the online as well as the literature search. All approaches of software-based business modelling were analyzed.

From a technical point of view, only tools with a desktop version or browser application were considered, since mobile applications such as BizCanvas [23] are less suitable for long-term planning of larger BM and 
depend heavily on the appropriate operating system. Furthermore, only tools with an English edition, which are available for free or have at least a free trial-version, were selected. The restriction to free tools enables comprehensive verifiability of the results. Even though the analysis of paid tools is also interesting, it leads to the problems of delimitation (e.g., some enterprise resource planning software also offers modules for business planning), incomplete findings and the poorer verifiability of the results. Basically, any drawing software could be used to create a visual model of a BM, but the focus of this analysis is on tools with specific functionality for business modelling. A tool has to provide at least the basic function of creating a BM using an underlying BM visualization method in order to be included in the results. Only tools that include a BM modeling method, such as the BMC [37] or the magic triangle [2], were selected. This ensures that the software is at least to some extent BM-specific and might offer BMI functionalities. If other methods were available within a tool that are suitable for BMI, these additional methods were only counted if a connection to the BM method is apparent to the user. Thus, in a pure method collection with no connection of methods for BMI, only the initial method for representing a BM counts for the evaluation of the BMI tool support. Other exclusion criteria are applications and tools that were no longer available.

\subsection{Tool comparison}

We analyzed the tools first in terms of how suitable they are for different BMI phases. To do this, we used a predefined standard BMI use case that allows to examine all tools with the same initial conditions. This use case was applied per tool by a group of researchers as far as it was possible in the respective tool. This use case supports the evaluation of the tools in such a way that it is predefined and thus covers BMI phases that either can be, cannot be, or can only partially be represented in the tools. A summary of the case is:

A fictitious company sells daily newspapers as print editions. The newspaper is aimed at the general population, but also has a large business section. Sales figures have been stagnating for some time and the company wants to change its BM to develop new sales markets and generate more revenue. In addition, the company also wants to attract younger readers to the newspaper. Momentarily, the company does not plan to introduce a completely new product but would like to expand the newspaper, possibly through digital offerings. The company has a website with some news, but no actual digital format of the newspaper. The company's employees are basically familiar with the BMC method, but have little previous experience in its application or with subsequent structured steps to BMI. Long-term goals are the acquisition and retention of new customers and an increase in sales. A more detailed version of the case can be provided by the authors.

As many organizations face similar problems and the process of change or innovation is usually rather informal, it is even more interesting to analyze how software can be of assistance. Although current software may be outdated in a few months, the criteria below can be used to examine software for its utility for BMI and to identify gaps in existing research. Criteria for the BMI process were derived from the process described in section 2.1. Wirtz defines sub-aspects for each category [8] which are used to check the coverage of the BMI phase respectively within a tool:

- Analysis of initial situation: representing the current status of a BM or creating a new BM; it must be possible to model certain BM elements like product/service, customers, market/competition

- Idea generation: presence of methods for generating customer insights, scenarios, storytelling or tool-based idea generation

- Feasibility analysis: depicting or describing the business environment, like competitors or legal regulations, analysis of interdependencies in the BM

- Prototyping: analysis and comparison of different BMI alternatives, possible development and refinement of several BMI concepts

- Decision-making: evaluating each BMI alternative, realization and test of the BMI, support for the selection of one BMI design, possible adaptions

- Implementation: support for an implementation plan, team setup, stepwise realization description, implementation completion

- Monitoring: monitoring the BM performance, controlling the value proposition, deriving implications for adapting the BM respectively the BMI idea

- Securing Sustainability: potential adaption of the BMI associated with organization-wide learning, securing competitive advantages, isolating mechanism towards competitors

The second set of criteria addresses different modelling scenarios which are closely linked to requirements in the BMI process and have a more distinct focus on BM designers [28]. The modelling scenarios provide information about which tool can be used for which problem or objective, and for which cases the software support for modelling can be expanded:

- Developing a BM from scratch

- Analyzing a BM to discover adaption and innovation potentials (and their execution)

- Analyzing the performance of an existing BM

- Analyzing BM in general for idea generation 
Every criterion can be either fulfilled, partly fulfilled or not fulfilled. In case of "analysis of initial situation" for example, there has to be the possibility to describe or depict the current status of the BM. If aspects, such as products or target groups can be modelled, the criterion is fulfilled. If only some BM elements are offered, but e.g., cost or revenue models are missing, the criterion is only partly fulfilled. This assessment was carried out for all BMI phases and modelling scenarios using the standard BMI use case. The procedure was conducted by the authors of this paper independently to uncover initial similarities. Subsequently, discrepancies and their reasons were discussed to ensure a more objective evaluation. In each case, attempts were made to find a consensus on the critical points that best reflect the features and capabilities of the software tool as well as to identify weaknesses and critical points within the research methodology. In all ambiguous cases one iteration was sufficient to find consensus.

\section{Findings}

\subsection{Overview}

By conducting the online search, a total of 16 relevant applications for modelling and innovating BM could be identified (table 2). All accessible tools from the literature search could be found as well. Unfortunately, seven of the tools presented by Szopinski et al. [23] were no longer available.

Overall, it is evident that the larger part of the BMI phases as well as the modeling scenarios are not covered. In particular, the more complex later phases are not or hardly supported by software. Three tools (Archi, e3value networked BM, Miro) do not use the BMC as the underlying method or offer at least one alternative to it. Using another modelling language in these cases allows the tools to partially map more information to the $\mathrm{BM}$, as relationships between elements of the BM can be modeled and weighted. However, the idea of such element relations is also adopted by tools that use the BMC method (7). The tools found have different orientations and can be roughly divided into three categories: pure BM representation $(1,2,4,5,13)$, online method collections/whiteboards $(8,9,10,11,15,16)$, dedicated BMI or strategy tools $(3,6,7,14)$. The extent to which the BMI phases and modelling scenarios are supported is described below.

\subsection{Tool support for different BMI phases}

The results in the area of BMI-support show a clear picture. The majority of tools offers the design and representation of BM, while only a few support the users in prototyping, decision-making or implementation of their BM (figure 3). Partial support of a phase was also counted for the BMI phases, so that these tools are shown in the figure as equivalent to those that provide full support. In the following, we will take a closer look at some of the specifics and differences in the support of the BMI phases and thus transformation efforts:

- Analysis of initial situation: Although all tools support this phase, as they can be used to create an initial BM, the support during initial modeling by descriptions or user guidance varies across the tools.

- Idea generation: Here, the online method collections perform particularly well, as they all have various software-supported idea generation methods linked to the BM. None of the tools makes suggestions based on the user's input, e.g., by using natural language processing algorithms. One tool (14) uses the ideas to generate proposals for the $\mathrm{BM}$ based on BM patterns. It is stated that the data can be matched with similar BM within the tool's database to generate suggestions.

- Feasibility analysis: In particular, the tools that enable connections between elements $(6,7)$ can support this step well. By modeling financial flows, for example, competitors or market barriers can be represented as separate nodes with relationships to and influence on other elements.

- Prototyping: Different BM can be modeled per tool, but no tool offers the possibility to directly contrast BM or their versions to highlight differences and similarities e.g., by color. In this phase, version histories could be used. An exception is (7), which supports the dedicated creation of scenarios, especially for the estimation of financial impacts.

- Decision-making: This phase receives very little software support, probably because it involves a strategic decision for or against a BMI, which is more related to the direction of an organization than to algorithmic solution finding. However, this step is also about evaluating and testing a BM, with two tools showing approaches to support these activities $(3,14)$. (14) offers the possibility to create an "experiment" for each BM with assumptions, the experiment and a decision, which allows at least a tool-supported documentation of the decision-making process. (3) is the only tool that provides the user with paths for different BMI scenarios using various methods, each suitably linked for the user's BMI objective. The tool offers in addition to the analysis of financial ratios, a so-called BM stress test [20], [21], which can be used to illustrate future developments and risks. 
Table 2: Tool support for BMI and modelling scenarios

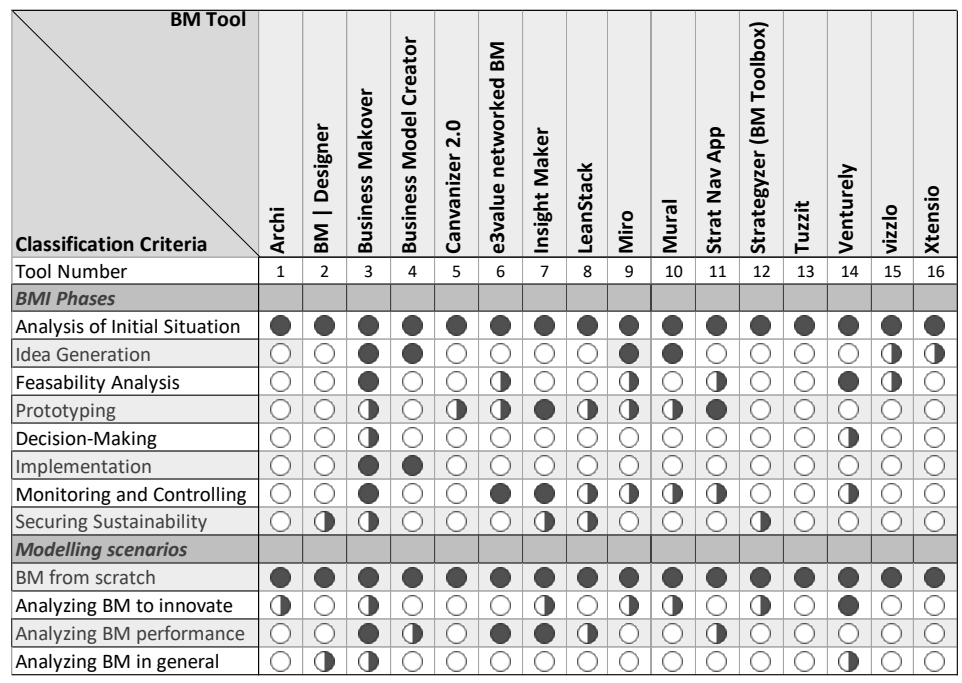

\begin{tabular}{l|l}
\hline $\mathbf{N r}$ & Reference \\
\hline $\mathbf{1}$ & https://www.archimatetool.com \\
\hline $\mathbf{2}$ & https://bmdesigner.com \\
\hline $\mathbf{3}$ & $\begin{array}{l}\text { https://www.businessmakeover.eu } \\
\text { https://webtools.innovalor.nl/\#/bmc }\end{array}$ \\
\hline $\mathbf{4}$ & $\begin{array}{l}\text { https://howtostartanllc.com/business- } \\
\text { center/business-model-canvas }\end{array}$ \\
\hline $\mathbf{5}$ & https://canvanizer.com \\
\hline $\mathbf{6}$ & https://www.e3value.com \\
\hline $\mathbf{7}$ & https://insightmaker.com/tag/Business- \\
\hline $\mathbf{8}$ & Model \\
\hline $\mathbf{9}$ & https://leanstack.com \\
\hline $\mathbf{1 0}$ & https://miro.com \\
\hline $\mathbf{1 1}$ & https://mural.co \\
\hline $\mathbf{1 2}$ & https://www.stratnavapp.com \\
\hline $\mathbf{1 3}$ & https://strategyzer.com/app \\
\hline $\mathbf{1 4}$ & https://app.venturely.io \\
\hline $\mathbf{1 5}$ & https://vizzlo.com \\
\hline $\mathbf{1 6}$ & https://xtensio.com/toolbox/strategy \\
\hline & \multicolumn{2}{|}{ fulfilled $\quad$ partly fulfilled $\quad$ not fulfilled } \\
\hline
\end{tabular}

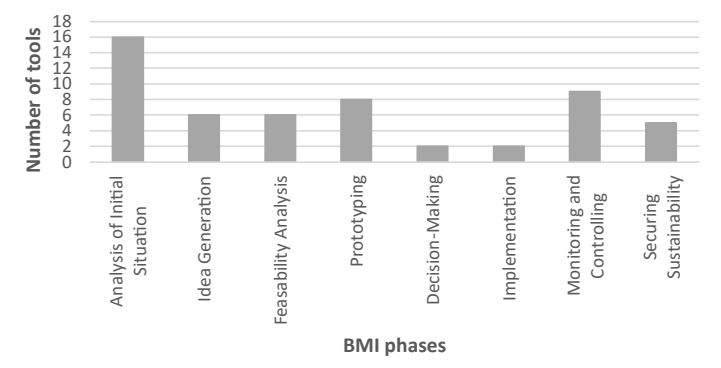

Figure 3: Tool support across the BMI process

- Implementation: The only support available across the tools was the possibility to create an implementation plan.

- Monitoring: Again, especially tools with connections and weightings between elements or nodes allow conclusions and controlling. The majority of tools uses the input of financial values for a monitoring, such as return on investment (ROI) monitoring. Some tools even offer a simulation functionality $(6,14)$. It should be noted that users may not be willing to disclose internal financial metrics to an online tool. This argument does not apply to the tools with desktop versions that run locally.

- Securing Sustainability: Basically, none of the tools supports this phase. However, since one subcategory for the phase is learning and sharing of the gained knowledge, the tools that make methodological knowledge accessible and applicable to users and allow a team of editors to work on the project received a "partially fulfilled". Some tools even offer video training on BMI and further steps during and after the tool use.
Only two of the tools found $(3,14)$ guide the user through a BMI process by means of questions, steps or methods that build on each other. All other tools support the BMI phases either by having different methods belong to one tool menu, or they are not linked at all for the user $(9,10)$ and must be linked manually, which would require method knowledge.

\subsection{Tool support for different modelling sce- narios}

Analogous to the BMI process, support for different approaches to a BMI project is considered below in terms of modeling scenarios:

- Developing a BM from scratch: Even though each tool is capable of representing the BM, the tools could be evaluated differently depending on the user's experience. Many tools require methodological knowledge, e.g., the BMC method, while others provide step-by-step instructions, question-based approaches or video tutorials for creating a BM.

- Analyzing a BM to discover adaption and innovation potentials: Most tools provide analysis capabilities through additional methods, such as swot (strengths, weaknesses, opportunities, and threats) or pestle (political, economic, social, technological, legal, environmental factors). However, in doing so, the modeler must adjust to a new method and the inputs can be independent of the initial BM input. An advantage is offered by the realizations which embed relationships in the BM because the user sees all dependencies and inputs at a glance and the interlocking with the BM still exists. 
- Analyzing the performance of an existing BM: Conclusions about a currently used BM can at most be derived via the analysis functions from the previous modeling scenario. The possibility of drawing conclusions about a BM via customer assessments or internal process data, for example, is not supported by any of the tools. In all analysis scenarios, a connection to an organization-wide software tool or a broader database could open up further possibilities for BMI support but is difficult to implement in a single BM tool.

- Analyzing BM in general: This scenario is achieved by the tools in two ways. Some tools $(2,3,12,14)$ have sample BM from well-known companies, and partly use them to explain how to create a BM. However, these are usually only three to four company examples per tool. There is no possibility to sort the examples by industry or similar elements With the exception of one tool (12). A collection of BM, that could be filtered by industry, BM pattern, or value proposition could be used both to generate ideas and to make recommendations. One tool (14) pursues the database approach to some extent, as it uses data of already created BM to propose BM patterns matching the user's inputs.

In the context of the modeling scenarios, special functionalities of the tools that go beyond the pure initial modeling have been identified. A set of tools provides knowledge about BMI in the form of videos, textual or interactive method descriptions or BM examples $(2,3$, $7,8,9,12,14)$. Connected with learning about methods is the user guidance that helps users to perform steps in the BMI process. This is achieved either through questionnaire-like approaches or by combining different methods to a single BM kit or bundle to make the connection between the methods apparent $(3,9,10)$. Analysis functionalities can be divided into the subsections financial aspects $(3,6,7,14)$, environmental influences $(3,10,11,14)$ and general evaluation of a BM idea (3, $6,8,11,12$ ). For this purpose, the tools provide e.g., ROI calculators, competitor lists, swot analyses or rulechecking. In terms of recommendations for BMI, as mentioned above, only one tool (14) uses a database of already created BM to match BM patterns to the own BM. Other tools refer to paid courses or consulting services $(3,8,12)$.

In total, modeling of BM performance and concrete incorporation of analysis results into BM modeling do not seem to be widespread yet. The use of BM examples is rudimentarily used within the tools.

\subsection{Addressing the research questions}

Summing up, with respect to RQ1 (Which software tools for BM exist and how can they be assed?) the above-described analysis shows the following: There exist several tools with different focuses in terms of BM support. On the one hand these are tools for the pure presentation of $\mathrm{BM}$, online method collections that also integrate BM and furthermore tools that are specifically about BM development and evolution. The individual BMI phases with their sub-categories seem suitable for evaluating BM software tools. For a better comparability, the BMI newspaper use case was represented as far as possible within a tool in order to assess its BMI support. Concerning RQ1a (To what extent do these tools support the phases of BMI?) our results suggest that early phases of the BMI process are supported by the majority of tools, as the strategic relevance as well as the uncertainty and risks increase over the phases. RQ1b (In which way do the tools offer the support for the user?) cannot be answered in a general way, because many tools offer unique features for BMI and modelling scenarios. This circumstance implies that for the BMI process, different functions and methods are suitable for achieving a target or that different targets entail a different focus of the tools.

Addressing RQ2 (Which modelling scenarios are covered with the help of the BM software tools?), our results show that creating an initial BM or making simple textual adaptions to an existing model can be accomplished within all found BM software tools. The analysis of possible shortcomings as well as recommendations and modelling assistance are not yet strongly developed. Using existing BM as a basis for modeling support seems promising but is hardly implemented so far. One reason for this could also be the need for a larger $\mathrm{BM}$ database. This assessment is reinforced by comparing statements from literature with the BMI tools (e.g., [14], [15]). Research suggests, for example, to use BM patterns [2] for innovation, but this has so far only been implemented in one of the found tools.

\section{Discussion and Implications for Soft- ware-supported BMI}

The results reflect that there are many efforts to support BMM and BMI on the software side to facilitate transformation processes and make existing knowledge accessible and usable. No definitive answer can be given to the question of why later phases are less supported by software, as various reasons can play a role. On the one hand, it is possible that appropriate methods and tool implementations have not yet emerged. On the other hand, the optimization and innovation of BM with software is 
more difficult to implement than, for example, process optimization. Processes can be recorded, evaluated and checked for consistency as well as syntactic and semantic correctness. Relevant key figures such as cycle times or costs can be analyzed and, if necessary, improved. While processes can be simulated and checked with relatively little effort, testing BM is far more complex. In business processes clear cause-effect-relationships can be found and decisions can be made by measuring key figures. For BMI, this is hardly possible because different and partly unknown variables could matter. Phases such as "secure sustainability" are more difficult to represent by software than a pure BM visualization. Therefore, not every BMI phase may necessarily need to be fully supported by software. Many steps within the BMI process also require the observation over a longer period of time, which is still little considered, since mostly only static images of a BM at a point in time are depicted, but not dynamic changes over time.

None of the tools found offers a deep integration with business activity monitoring or data management systems. Especially for the phases of controlling and implementation, one core question is, if $\mathrm{BM}$ aspects with complex underlying processes can really be covered in a single BM tool, or if such a tool needs integration points with existing IT solutions to gather the required data from. It should also be noted that software in the $\mathrm{BM}$ area is not meant to make fully automated strategic decisions. It should rather show alternatives or give recommendations. Text mining methods could be used for this purpose to make the content of the BM as well as subsequent methods more usable. Besides, since BM are located one level above the business processes, an attempt could be made to establish a link between process monitoring and the BM level (see [38]).

Our approach is not free of limitations. In the analysis of software-support for BMI, we conducted a literature search as well as an online search for current tools. Although we cannot exclude the existence of further tools, the analysis already provides insights into existing difficulties, gaps and advantages of BMI software support. Since only freely available software was considered, no statement can be made about the scope or potential of tools that require charges. However, since consulting services were found within the online search, it is questionable whether tools exist that go far beyond the scope of the freely available ones. The criteria of the tool evaluation can be examined in more detail in a next step, in order to emphasize the exact design of sub-aspects more precisely. A user study, e.g., in the context of a company example, could provide information about the perceived benefits of individual tools and their functions for BMI. In addition, the extent to which prior knowledge of BMI methods is assumed can be investigated more closely.
At the current stage, BM tools can support BM modelers by leading through the different BMI phases step-by-step and linking suitable methods for the user's objectives. The requirements and relevant data of the user would then have to be collected, for example by means of a questionnaire. Some tools already pursue such an approach. In our investigation, tool (3) supported the most phases of the BMI process. It should be noted that the criteria of our tool analysis were derived from the literature and (3) also includes content and especially methods from literature as the tool was developed as part of a research project. In this respect, it can be argued that an investigation according to different criteria, e.g., from practitioners, may lead to different findings. That many online method collections have relatively high coverage of the BMI process is due to their wide range of methods. It is probably not the sole aim of a method collection tool to provide targeted support for BMI, but it does provide users with methods that can be meaningfully employed within a BMI project if offered in one view. The variety of methods available in the BM tools can be seen as a possible evidence for the difficulty of representing creative processes with complex and strategically long-term goals by software. Therefore, it can also be learned from the analysis that a connection of methods might be necessary and that a BMI is difficult to model from start to finish with just a single method.

The analyzed BM tools show first indications for becoming more adaptive in the future and offering integration points for enterprise software or vice versa. This could also bypass problems such as the transfer of sensitive company data to third parties and helps to depict and trace the BMI process within an organization. Initial approaches in this area already exist [39] but need further development. Moreover, extending the scope of BMI to technology-focused approaches might be beneficial to foster technology-driven innovation in the BM design process.

\section{Conclusion}

This contribution presents an analysis of software support for the BMI process and associated modeling scenarios. For this purpose, we have introduced criteria and a standard use case for evaluating existing software tools. Besides this reusable approach for BM modelers, we have identified existing gaps within software support for business modeling, as well as possible causes and solutions. Our analysis identified existing approaches for tool-based business modelling in literature as well as online and evaluates them with regard to their level of support. The analysis showed that early phases of the BMI process are well supported by software, while later phases, such as decision-making or controlling, are only 
poorly supported. Possible reasons for this are the increasing complexity and uncertainty associated with later stages of BMI. However, several tools and literature already show approaches to support these phases, in order to achieve a more holistic business modeling approach. Furthermore, collections of existing BMs for modeling and ideation support seem particularly promising.

It may also not be sufficient to base all BMI phases to a single (or multiple) $\mathrm{BM}$ representation alone. In many cases other methods, not directly related to BM, are used for the innovation process. Their expedient linking and enrichment with data has been little considered so far and should be further investigated. The current software support already offers BM designers and those responsible for transformation good approaches to using and passing on existing BM knowledge. The evaluation scheme presented in this work can also be used to quickly assess the suitability of tools developed in the future for different modeling scenarios and objectives. The tools as well as the underlying methods will probably have to be further developed to be able to usefully support more complex phases of BMI and the associated modeling scenarios.

Acknowledgements: The research presented in this paper has been carried out within the Mittelstand-Digital Zentrum Kaiserslautern funded by the Federal Ministry for Economic Affairs and Energy (BMWi). We further like to thank the reviewers for their comments which have helped to improve this contribution.

\section{References}

[1] M. Heikkilä and H. Bouwman, "Business Model Innovation in European SMEs - Descriptive analysis of quantitative survey and case survey data," 31 st Bled eConference: Digital Transformation: Meeting the Challenges, BLED 2018, no. June, pp. 543-560, 2018.

[2] O. Gassmann, K. Frankenberger, and M. Csik, The Business Model Navigator: 55 Models That Will Revolutionise Your Business. Harlow et al.: Pearson Professional, 2014.

[3] B. W. Wirtz, Digital Business Models - Concepts, models, and the Alphabet Case Study. Springer International Publishing, 2019.

[4] A. Osterwalder, Y. Pigneur, and C. Tucci, "Clarifying business models: Origens, present, and future of the concept," Comunications of AIS, vol. 16, no. July, pp. 1-25, 2005.

[5] J. S. Schwarz and C. Legner, "Business model tools at the boundary: exploring communities of practice and knowledge boundaries in business model innovation," Electronic Markets, vol. 30, no. 3, pp. 421-445, 2020.
[6] B. Fritscher and Y. Pigneur, "Computer Aided Business Model Design: Analysis of Key Features Adopted by Users," 2014 47th Hawaii International Conference on System Sciences, pp. 3929-3938, 2014.

[7] T. Burkhart, D. Werth, J. Krumeich, and P. Loos, "Analyzing the Business Model Concept - A comprehensive classification of Literature," in Thirty Second International Conference on Information Systems, 2011.

[8] B. W. Wirtz, "Business model management : design - instruments - success factors." Wiesbaden : Gabler, 2011.

[9] B. Demil and X. Lecocq, "Business model evolution: In search of dynamic consistency," Long Range Planning, vol. 43, no. 2-3, pp. 227-246, 2010.

[10] C. Zott, R. Amit, and L. Massa, "The business model: Recent developments and future research," Journal of Management, vol. 37, no. 4, pp. 1019-1042, 2011.

[11] H. Chesbrough, "Business Model Innovation : Opportunities and Barriers," Long Range Planning, vol. 43, no. 2-3, pp. 354-363, 2010.

[12] B. W. Wirtz and P. Daiser, "Business Model Innovation Processes: A Systematic Literature Review," Journal of Business Models, vol. 6, no. 1, pp. 40-58, 2018.

[13] J. Webster and R. T. Watson, "Analyzing the Past to Prepare for the Future: Writing a Literature Review.," MIS Quarterly, vol. 26, no. 2, pp. xiii-xxiii, 2002.

[14] D. Szopinski, T. Schoormann, T. John, R. Knackstedt, and D. Kundisch, "How software can support innovating business models: A taxonomy of functions of business model development tools," in America's Conference on Information Systems: A Tradition of Innovation AMCIS, 2017, no. August, pp. 1-10.

[15] T. Schoormann, D. Behrens, and R. Knackstedt, "The noblest way to learn wisdom is by reflection: Designing software tools for reflecting sustainability in business models," in International Conference on Information Systems 2018, ICIS 2018, 2018, no. December.

[16] D. Szopinski, "Jumping, dumping, and pumping: Three mental principles for idea generation to activate softwarebased tools in business model innovation," 32nd Bled eConference Humanizing Technology for a Sustainable Society, BLED 2019 - Conference Proceedings, no. June, pp. 367-385, 2020.

[17] M. Voigt, R. Plattfaut, K. Ortbach, A. Malsbender, B. Niehaves, and J. Becker, "Evaluating business modeling tools from a creativity support system perspective - Results from a focus group in the software development industry," Proceedings - Pacific Asia Conference on Information Systems, PACIS 2013, 2013.

[18] K. Rambow-Hoeschele et al., "Creation of a Digital Business Model Builder A Concept to Simulate a Digital Twin of a Business Model and Its Imperative Nature," 2018 IEEE International Conference on Engineering, Technology and Innovation (ICE/ITMC), pp. 1-7, 2018.

[19] M. Schröder, B. Falk, and R. Schmitt, "Evaluation of Cost Structures of Additive Manufacturing Processes Using a New Business Model," Procedia CIRP, vol. 30, pp. 311-316, 2015.

[20] H. Bouwman, J. Heikkilä, and M. Heikkilä, “Achieving agility using business model stress testing," pp. 149-162, 2018. 
[21] T. Haaker, H. Bouwman, W. Janssen, and M. De Reuver, "Business model stress testing A practical approach to test the robustness of a business model," Futures, vol. 89, no. April, pp. 14-25, 2017.

[22] G. Peinel, M. Jarke, and T. Rose, "Business models for eGovernment services," Electronic Government, an International Journal, vol. 7, no. 3, pp. 380-401, 2010.

[23] D. Szopinski, T. Schoormann, T. John, R. Knackstedt, and D. Kundisch, "Software tools for business model innovation: current state and future challenges," Electronic Markets, vol. 30, pp. 469-494, 2020.

[24] I. Meyer, R. Hammerschmidt, L. Kubitschke, and S. Müller, "How to Make a Service Sustainable? An Active Learning Simulation Approach to Business Model Development for Integrated Care BT - Handbook Integrated Care," V. Amelung, V. Stein, N. Goodwin, R. Balicer, E. Nolte, and E. Suter, Eds. Cham: Springer International Publishing, 2017, pp. 277-294.

[25] P. Johnson, M. E. Iacob, M. Välja, M. van Sinderen, C. Magnusson, and T. Ladhe, "Business Model Risk Analysis: Predicting the Probability of Business Network Profitability BT - Enterprise Interoperability," 2013, pp. 118-130.

[26] S. Boßelmann and T. Margaria, "Domain-Specific Business Modeling with the Business Model Developer BT Leveraging Applications of Formal Methods, Verification and Validation. Specialized Techniques and Applications," 2014, pp. 545-560.

[27] T. Schoormann, M. Stadtländer, and R. Knackstedt, "Designing business model development tools for sustainability - a design science study," Electronic Markets, 2021.

[28] M. Schief, Business Models in the Software Industry: The Impact on Firm and M\&A Performance. Wiesbaden: Springer Fachmedien Wiesbaden, 2014.

[29] C. Di Valentin, A. Emrich, D. Werth, and P. Loos, “Architecture and implementation of a decision support system for software industry business models," in 19th Americas Conference on Information Systems, AMCIS 2013, 2013, vol. 1, pp. 472-482.
[30] P. Ebel, U. Bretschneider, and J. M. Leimeister, "Leveraging virtual business model innovation: a framework for designing business model development tools," Information Systems Journal, vol. 26, no. 5, pp. 519-550, 2016.

[31] B. Kijl and D. Boersma, "Developing a business model engineering \& experimentation tool - The quest for scalable lollapalooza confluence patterns," in AMCIS 2010 Proceedings, 2010, vol. 662, pp. 78-80.

[32] T. John, D. Kundisch, and D. Szopinski, "Visual Languages for Modeling Business Models: A Critical Review and Future Research Directions," ICIS 2017: Transforming Society with Digital Innovation, no. December, 2017.

[33] D. Dellermann, N. Lipusch, P. Ebel, and J. M. Leimeister, "Design principles for a hybrid intelligence decision support system for business model validation," Electronic Markets, vol. 29, no. 3, pp. 423-441, 2019.

[34] A. Athanasopoulou and M. de Reuver, "Designing business model tooling for business model exploration: An experimental design for evaluation," 31st Bled eConference: Digital Transformation: Meeting the Challenges, BLED 2018, no. June, pp. 477-490, 2018.

[35] B. Fritscher and Y. Pigneur, "Task Models and Diagrams for User Interface Design," Task Models and Diagrams for User Interface Design, vol. 5963, no. July 2014, pp. 28-43, 2010.

[36] B. W. Wirtz, Business Model Mangement: Design - Instruments - Success Factors, 1st ed. Wiesbaden: Gabler, 2011.

[37] A. Osterwalder and Y. Pigneur, Business model generation, vol. 53, no. 9. Hoboken, NJ, USA: Wiley, 2010.

[38] T. Schoormann, S. Hagen, J. Brinker, S. Wildau, O. Thomas, and R. Knackstedt, "Towards Aligning Business Models with Business Processes: A Tool-based Approach," Modellierung 2020, Lecture Notes in Informatics (LNI), pp. 13-27, 2020.

[39] B. Fritscher and Y. Pigneur, "A Visual Approach to Business IT Alignment Between Business Model and Enterprise Architecture," Int. J. Inf. Syst. Model. Des., vol. 6, no. 1, pp. 1-23, 2015. 